\title{
Nerves Paralysises Associated to Nasal Obstruction: What is Your Diagnosis??
}

\author{
Ilham Rkain ${ }^{1,2 *}$, Safaa Touihmi ${ }^{1}$, Rachidi Alaoui Siham ${ }^{1,3}$ and Hicham mimouni ${ }^{4}$ \\ ${ }^{1}$ Department of otorhinolaryngology, Head and Neck Surgery, university hospital, Tangier, Morocco \\ ${ }^{2}$ Faculty of Medicine and Pharmacy of Tangier, Abdelmalek Saadi University, Tétouan, Morocco \\ ${ }^{3}$ Department of radiology, university hospital, Tanger, Morocco \\ ${ }^{4}$ Department of otorhinolaryngology, Head and Neck Surgery, Al kortobi hospital, Tangier, Morocco
}

*Corresponding author: Rkain Ilham, Department of otorhinolaryngology, Head and Neck Surgery, university hospital, Tangier, Faculty of Medicine and pharmacy of tangier, Abdelmalek Saadi University, Tétouan, Morocco.

Received Date: August 07, 2020

Published Date: September 01, 2020

\section{Case Report}

A 60-year-old man presented with complaints of nasal obstruction (left side), smell disorder, headache and neuralgia on the left side of his face for 2 years, ptosis and diplopia followed by reduction vision of the left side. The patient was treated in another institution with antibiothiques and antituberculous drugs. After two weeks, the treatment was stopped because of the deterioration of the neurologic status, and the appearance of new symptoms such as mouth opening limitation, dyspnea, difficulty of swallowing (dysphagia) and cerebellar syndrome. So, all cranial nerves at the left side were dysfonctionnal. At the onset of these symptoms, tomography computed of the head was done, what is your diagnosis??

(Figure 1,2) Presence of a large heterogeneous $9 \mathrm{~cm}$ tumor of the nasopharynx and the left nasal fossa, extended to the maxillary sinus, oropharynx, skull base, brainstem and to the left cerebellar hemisphere. Nasal endoscopy biopsy shows a UCNT of the cavum.

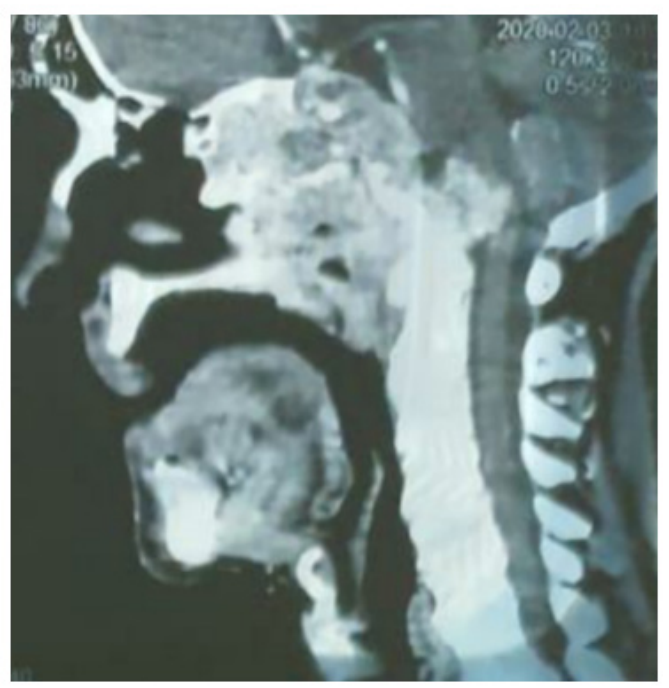

Figure 1: Sagital CT scan passing through the nasal cavity. 


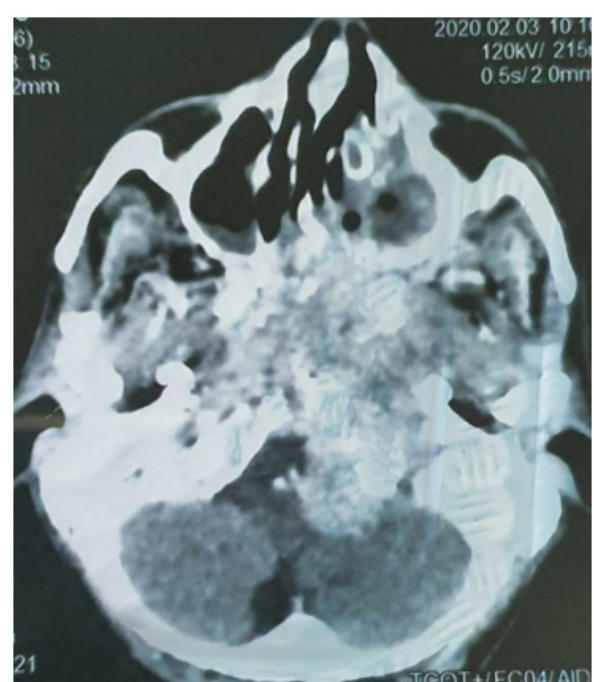

Figure 2: Axial CT scan passing through the nasopharynx.

\section{Discussion}

Guillain Alajouanine Garcin syndrome or garcin syndrome is rare disorder with progressive unilateral involvement of all or at least 7 cranial nerves, first reported in 1926 [1]. This syndrome is frequently seen in the tumors of nasopharynx [2,3] and the base of the skull, which does not affect the brain itself. The syndrome is progressive and, in its complete form, is seen only very rarely. Some reported cases have other symptoms such as headache such as our case. Prognosis as a rule is unfavorable. The findings on the CT scan and MRI scan are important in early diagnosis [4]. Many cases of Garcin's syndrome have been reported. These include cases that are caused by tonsillar carcinoma, carcinomatous leukemic meningitis, and para-nasal and parotid tumors. Nasopharyngeal carcinoma frequently involves cranial nerves because of its proximity to the skull base $[4,5]$.

\section{Conclusion}

This case illustrates the need to consider nasopharyngeal carcinoma as the most common cause of garcin syndrome in the presence of unilateral cranial pair involvement associated with olfactory disorders.

\section{Acknowledgement}

None.

\section{Conflict of Interest}

No conflict of interest.

\section{References}

1. Guillan R AT, Garcin R (1926) Global unilateral paralytic syndrome of the cranial nerves. Bull Med Hop (Paris) 50: 456-460.

2. Goel A, Bhayani R, Satoskar A, Nagpal RD (1995) Massive nasopharyngeal carcinoma mimicking Garcin syndrome. Neurol India 43(2): 120121.

3. Guillain G, Alajouanine Th, Garcin R (1926) Global unilateral paralytic syndrome of the cranial nerves. Bull Med Hop (Paris) 50: 456.

4. Barbara Casolla, Serena Candela, Antonio Ciacciarelli, Ludovico Ciolli, Andrea Romano, et al. (2019) Relapsing Long-Lasting Garcin Syndrome Revealing Skull Base Diffuse B Cell Lymphoma: The Diagnosis through the "Hartel's Route" J Neurol Surg B Skull Base 80: 105-108.

5. F Letournel (2004) Garcin syndrome revealing non-Hodgkin's malignant lymphoma. Rev Neurol (Paris) 160(10): 952-955. 